OPEN ACCESS

Edited by:

Lionel Carneiro,

The Ohio State University,

United States

Reviewed by:

Christelle Le Foll,

University of Zurich, Switzerland

Céline Cruciani-Guglielmacci,

Université Paris Diderot, France

*Correspondence:

Erika S. Piedras-Rentería epiedra@/uc.edu

tPresent address:

Paula P. Perissinotti,

Instituto de Fisiología, Biología Molecular y Neurociencias (IFIBYNE, UBA-CONICET), Ciudad Universitaria,

Buenos Aires, Argentina

Elizabeth Martínez-Hernández,

Department of Physiology

and Biophysics, Rush University,

Chicago, IL, United States

¥ORCID:

Paula P. Perissinotti orcid.org/0000-0002-9757-0610

Elizabeth Martínez-Hernández orcid.org/0000-0002-5004-2239

Erika S. Piedras-Rentería

orcid.org/0000-0001-7983-585X

Specialty section:

This article was submitted to Neuroenergetics, Nutrition and Brain

Health,

a section of the journa

Frontiers in Neuroscience

Received: 10 March 2021

Accepted: 30 April 2021

Published: 11 June 2021

Citation:

Perissinotti PP,

Martínez-Hernández E and

Piedras-Rentería ES (2021)

TRPC1/5-Cav3 Complex Mediates Leptin-Induced Excitability

in Hypothalamic Neurons.

Front. Neurosci. 15:679078.

doi: 10.3389/fnins.2021.679078

\section{TRPC1/5-Cav3 Complex Mediates Leptin-Induced Excitability in Hypothalamic Neurons}

\author{
Paula P. Perissinotti ${ }^{\ddagger \neq}$, Elizabeth Martínez-Hernández ${ }^{\ddagger \ddagger}$ and Erika S. Piedras-Rentería ${ }^{* \neq}$ \\ Cell and Molecular Physiology Department and Neuroscience Division of the Cardiovascular Research Institute, Loyola \\ University Chicago, Maywood, IL, United States
}

Leptin regulates hypothalamic $\mathrm{POMC}^{+}$(pro-opiomelanocortin) neurons by inducing TRPC (Transient Receptor Potential Cation) channel-mediate membrane depolarization. The role of TRPC channels in POMC neuron excitability is clearly established; however, it remains unknown whether their activity alone is sufficient to trigger excitability. Here we show that the right-shift voltage induced by the leptin-induced TRPC channelmediated depolarization of the resting membrane potential brings T-type channels into the active window current range, resulting in an increase of the steady state T-type calcium current from 40 to $70 \%$ resulting in increased intrinsic excitability of POMC neurons. We assessed the role and timing of T-type channels on excitability and leptin-induced depolarization in vitro in cultured mouse POMC neurons. The involvement of TRPC channels in the leptin-induced excitability of POMC neurons was corroborated by using the TRPC channel inhibitor 2APB, which precluded the effect of leptin. We demonstrate T-type currents are indispensable for both processes, as treatment with NNC-55-0396 prevented the membrane depolarization and rheobase changes induced by leptin. Furthermore, co-immunoprecipitation experiments suggest that TRPC1/5 channels and Cav3.1 and Cav3.2 channels co-exist in complex. The functional relevance of this complex was corroborated using intracellular $\mathrm{Ca}^{2+}$ chelators; intracellular BAPTA (but not EGTA) application was sufficient to preclude POMC neuron excitability. However, leptin-induced depolarization still occurred in the presence of either BAPTA or EGTA suggesting that the calcium entry necessary to self-activate the TRPC $1 / 5$ complex is not blocked by the presence of BAPTA in hypothalamic neurons. Our study establishes T-type channels as integral part of the signaling cascade induced by leptin, modulating POMC neuron excitability. Leptin activation of TRPC channels existing in a macromolecular complex with T-type channels recruits the latter by locally induced membrane depolarization, further depolarizing POMC neurons, triggering action potentials and excitability.

\section{Keywords: $\mathrm{Ca}_{v}$ 3.1, $\mathrm{Ca}_{v}$ 3.2, TRPC channel, hypothalamus, leptin, POMC}

\footnotetext{
Abbreviations: AgRP, agouti-related peptide; AP, action potential, DIV, days in vitro; KLHL1, Kelch-like 1; LRb, Leptin receptor; LVA, low-voltage activated; NPY, neuropeptide Y; PIR, post-inhibitory rebound; POMC, proopiomelanocortin positive neurons; TRPC, Transient Receptor Potential Cation; TRPC, Transient Receptor Potential Cation.
} 


\section{INTRODUCTION}

Leptin regulates energy homeostasis and serves as a satiety afferent signal in the homeostatic control of adipose tissue mass (Schwartz et al., 2000; Harvey and Ashford, 2003), reducing food intake, increasing energy expenditure and regulating the reward value of nutrient (Ahima and Flier, 2000; Domingos et al., 2011; Williams and Elmquist, 2012). Leptin exerts its physiological action through its specific receptor (LRb), which is highly expressed in hypothalamus and other brain areas (Shioda et al., 1998). Leptin's effects on hypothalamic homeostatic feeding circuits are well established (Kenny, 2011); it negatively modulates orexigenic agouti-related peptide (AgRP)/neuropeptide Y (NPY) neurons by Kv2.1 channelmediated membrane hyperpolarization (Baver et al., 2014). In contrast, anorexigenic POMC positive neurons are depolarized by leptin (Cowley et al., 2001). This depolarization is mediated via a Jak2-PI3 kinase-PLC $\gamma$ pathway that ultimately activates TRPC channel activity (Qiu et al., 2010). Similarly, a subset of POMC neurons in the arcuate nucleus responsive to serotonin via the 5$\mathrm{HT}_{2 \mathrm{C}}$ receptor are also activated via TRPC channels, suggesting TRPC channels are a common signaling mechanism mediating anorexigenic signaling in the hypothalamus (Sohn et al., 2011). TRPC5 channels are also the molecular mediators of the acute leptin and serotonin effect in POMC neurons (Gao et al., 2017).

The role of TRPC channels in POMC neuron excitability is clearly established; however, it is not known whether their activity alone is sufficient to trigger excitability. Here we used cultured hypothalamic neurons from mice to characterize the role of T-type $\mathrm{Ca}^{2+}$ channels and leptin-induced POMC neuron excitability. Our data demonstrate T-type channels are necessary for POMC neuron excitability, by being involved in the excitatory cascade induced by leptin in these neurons. Blockade of either TRPC or T-type channel function prevents the effect of leptin on hypothalamic neuron excitability. Moreover, we demonstrate that: (a) TRPC1 and TRPC5 channels co-immunoprecipitate with T-type channels in the hypothalamus, (b) TRPC-T-type channel complexes exist in a functional microdomain, and (c) TRPC-induced depolarization in these domains triggers neuronal excitability via recruitment of T-type channels. Thus, this study confirms T-type channels constitute a target to modulate leptinactivated neurons and their functions, such as energy balance and food intake.

\section{MATERIALS AND METHODS}

The animal protocols used in this study were reviewed and approved by an independent Institutional Animal Care and Use Committee (IACUC 2016032). Mixed background $(129 \mathrm{~S} 1 / \mathrm{Sv}-\mathrm{Oca} 2+\mathrm{Tyr}+\mathrm{Kitl}+\mathrm{C} 57 \mathrm{BL} / 6) \mathrm{WT}$ and EGFP-POMC ${ }^{+}$mice $[129 \mathrm{~S} 1 / \mathrm{Sv}-\mathrm{Oca} 2+\mathrm{Tyr}+\mathrm{Kitl}+\mathrm{C} 57 \mathrm{BL} / 6-$ $\mathrm{Tg}$ (Pomc-EGFP)1Low/J] (The Jackson Laboratory, RRID:IMSR_JAX:009593) were fed on an ad libitum standard commercial pellet diet. No exclusion criteria were predetermined. Altogether, 12 newborn pups were used for hypothalamic cultures ( 1 pup per cell culture) and 17 adult mice (male) for immunoprecipitation experiments. The study was not pre-registered. Experiments were conducted in the afternoon.

\section{Neuron Cultures}

Whole hypothalamus were dissected from newborn mice (postnatal day 0, P0) and cultured as described in Perissinotti et al. (2014). Cells plated at a density of 25,000-35,000/coverslip and kept in a $5 \% \mathrm{CO}_{2}$ humidified atmosphere at $37^{\circ} \mathrm{C}$. Newborn pups (no sex determination) were killed by decapitation after cold-induced anesthesia, and their brains rapidly removed prior to hypothalamus dissection; each culture was generated from two independent mice, each plated on 6 coverslips (electrophysiology and ICC data was generated from at least three independent batches of cultures, $\mathrm{N}=6$ mice). Cryo-anesthesia abolished perception of pain of pups. This method has been validated by Veterinary Services to minimize animal suffering.

\section{Immunocytochemistry (ICC)}

WT neurons at $8-11$ DIV were prepared as described (Perissinotti et al., 2014). Primary antibody dilutions were: POMC 1:200 (Novus, RRID:AB_791643); AgRP, 1:50 (Sta. Cruz, RRID:AB_2258141), $\mathrm{Ca}_{V} 3.1$ 1:200 (Alomone, RRID:AB_2039779) and 1:50 (Millipore, Cat.\# MABN464), $\mathrm{Ca}_{V} 3.2$ 1:5 (supernatant, NeuroMab, RRID:AB_2069551) and 1:200 (Sta. Cruz, RRID:AB_2259537); TRPC1 (RRID:AB_2040234) $1: 100$ and TRPC5 (RRID:AB_2040241) 1:120 (Alomone). Secondary antibodies: Alexa-488 Goat anti-mouse (RRID:AB_2633275) and goat anti-rabbit (RRID:AB_143165), -594 goat anti-rabbit (RRID:AB_2762824), and -647 goat anti-chicken (RRID:AB_2762845), 1:2,000 (Molecular Probes, Eugene, OR). Image acquisition was done using a Olympus IX80 microscope, analyzed by deconvolution and processed with ImageJ freeware (NIH) (Schneider et al., 2012).

\section{Immunoprecipitation (IP)}

Adult mice $(24-30 \mathrm{~g})$ were deeply anesthetized with $2 \%$ Isofluorane, then quickly decapitated; whole brains or hypothalamus were isolated according to guidelines of the IACUC. At least two whole brains or two whole hypothalami were needed per $\mathrm{n}$ to assess the presence of complexes with $\mathrm{Ca}_{V} 3.1\left(\alpha_{1 G}\right)$ or TRPC1, whereas only one whole brain/one hypothalamus were needed per $\mathrm{n}$ for $\mathrm{Ca}_{V} 3.2\left(\alpha_{1 H}\right)$ or TRPC5 complexes; each experiment was replicated three times. Tissues were homogenized using a Bullet blender tissue homogenizer using $0.5 \mathrm{~mm}$ zirconium oxide beads (Next Advance) and spun down at $1,300 \times \mathrm{g}$ to eliminate debris. A fraction of the supernatant was reserved before immunoprecipitation and stored at $-80 \mathrm{C}$ until processing (input); the remaining volume was divided up in equal parts for all experiments. Samples were then processed by addition of the primary antibody and incubation for $1 \mathrm{~h}$ at $4^{\circ} \mathrm{C}$ (antibodies: $\alpha 1 \mathrm{H}$ (Santa Cruz Biotechnology, Inc., Santa Cruz, CA, United States, RRID:AB_2259537), $\alpha 1 \mathrm{G}$ [Millipore, Cat. \#MABN464); TRPC5 (Alomone, RRID:AB_2040241)], TRPC1 (Santa Cruz Biotechnology, Inc., Santa Cruz, CA, United States, RRID:AB_2207905), and IgG (Cat. \#20008-1-100 and 
\#20009-1-100, Alpha Diagnostics, San Antonio, TX) and overnight incubation, followed by incubation for $1 \mathrm{~h}$ with protein A/G agarose beads (Biovision, Mountain View, CA) on a shaking plate at $4^{\circ} \mathrm{C}$. The samples were washed and then precipitated in $0.1 \mathrm{M}$ glycine $\mathrm{pH} 3.5$ and neutralized with $0.5 \mathrm{M}$ Tris. $\mathrm{HCl}$ and $1.5 \mathrm{M} \mathrm{NaCl} \mathrm{pH} 7.4$ before SDS-PAGE electrophoresis $(8 \%$, at $100 \mathrm{~V}$ for $90 \mathrm{~min}$ ) followed by transfer to a PVDF membrane (BioRad). Membranes were washed in Tris-buffered saline (TBS) + Tween (TBST; 0.05\% Tween 20), blocked for $1 \mathrm{~h}$ in TBST $+5 \%$ milk at room temperature, and incubated at $4^{\circ} \mathrm{C}$ overnight with $\alpha 1 \mathrm{H}$ polyclonal $(1: 2,000)$, $\alpha 1 G$ monoclonal (1:500), TRPC1 monoclonal $(1: 1,000)$, or TRPC5 polyclonal $(1: 1,000)$ antibody. Incubation with goat anti-rabbit (RRID:AB_1185567) horseradish peroxidase (HRP)or anti-mouse (RRID:AB_228307) HRP-conjugated secondary antibodies was done at room temperature (1:2,000; Pierce). Blots were exposed to developing agent (Supersignal Femto Dura, Pierce) before analysis with a ChemiDoc MP System (BioRad).

\section{Electrophysiology}

Whole-cell patch clamp recordings were performed from cultured hypothalamic neurons from 8 to 11 DIV using an Axopatch 200B amplifier (Axon instruments, Union City, CA) at room temperature. Data were acquired at $1 \mathrm{kHz}$ and digitized at $20 \mathrm{kHz}$ using a Digidata $1322 \mathrm{~A}$ analog-to-digital converter. Pipettes pulled from borosilicate glass (Warner Instruments, Hamden, CT) had resistances of 3.5-4.5 M $\Omega$ when filled with intracellular solutions. Cells with series resistance $\left(R_{s}\right)<20$ $\mathrm{M} \Omega$ were used; $R_{s}$ was compensated online $(>80 \%)$. Data was acquired with and analyzed with pClamp 10 software (Molecular Devices). Cell capacitance was measured from a transient current evoked by a $5 \mathrm{mV}$ depolarizing step from a holding potential of $90 \mathrm{mV}$.

Calcium currents were recorded in an external solution containing (in mM) $5 \mathrm{CaCl}_{2}, 140$ TEA-Cl, 10 HEPES and 10 glucose $\left(\mathrm{pH} 7.4,300 \mathrm{mOsmol} / \mathrm{kgH}_{2} \mathrm{O}\right)$, using an intracellular solution containing (in mM) $108 \mathrm{CsMeSO}_{3}, 4 \mathrm{MgCl}_{2}, 10 \mathrm{Cs}$ EGTA, 9 HEPES, 5 ATP-Mg, 1 GTPLi and 15 phosphocreatineTris ( $\mathrm{pH} 7.4,290 \mathrm{mOsmol} / \mathrm{kgH}_{2} \mathrm{O}$ ). Voltage control was improved by increasing cell impedance using extracellular TEA and intracellular cesium to block $\mathrm{K}^{+}$conductances. I-V curve properties such as its negative slope and reversal potential were monitored for appropriate voltage control. For the study of calcium current properties, we avoided recording from neurons older than 10 DIV because the possibility of spaceclamp problems.

Resting membrane potential (RMP) and APs were recorded in external solution containing (in $\mathrm{mM}$ ) $135 \mathrm{NaCl}, 5 \mathrm{KCl}, 2 \mathrm{CaCl}_{2}$, $1 \mathrm{MgCl}_{2}, 10$ HEPES, 10 glucose ( $\mathrm{pH} 7.4,300$ mosmol $/ \mathrm{kgH}_{2} \mathrm{O}$ ) and intracellular solution containing (in $\mathrm{mM}$ ) $110 \mathrm{~K}$-gluconate, $20 \mathrm{KCl}, 2 \mathrm{MgCl}_{2}, 1$ EGTA, 10 HEPES, 2 ATP-Mg, 0.25 GTP-Li and 10 phosphocreatine-Tris ( $\mathrm{pH} 7.4,290$ mosmol/ $\mathrm{kgH}_{2} \mathrm{O}$ ). Drugs: NNC-550396 dihydrochloride (Cat. \#2268) and Leptin (Cat. \#2985) were purchased from Tocris (Bristol, United Kingdom).

Square protocols to obtain I-V curves and T-type steady-state activation and inactivation were done as described in Bean (1985) and Perissinotti et al. (2015).

\section{Current-Voltage Relationships (I-V Curves)}

Currents were elicited from a holding potential $\left(\mathrm{V}_{H}\right)=-90 \mathrm{mV}$ and depolarized for $150 \mathrm{~ms}$ to a test potential $\left(\mathrm{V}_{T}\right)=-70$ to +60 $\mathrm{mV}$, in $10 \mathrm{mV}$ increments.

\section{T-type Current-Rate of Membrane Potential Depolarization Relationship}

Different rates of membrane potential depolarization preceding the first action potential were obtained from hypothalamic neurons by changing the rate of current ramp stimulations (20-100 pA/s) in current clamp configuration: 40, 90, 180, 290, or $360 \mathrm{mV} / \mathrm{s}\left(\mathrm{V}_{H}=-75 \mathrm{mV}\right)$. These voltage templates were used in voltage clamp configuration to stimulate calcium currents. The membrane potential was held at a $\mathrm{V}_{H}$ of -50 or $-90 \mathrm{mV}$ before clamping the voltage at $-75 \mathrm{mV}$ for $10 \mathrm{~ms}$ to run the stimulation protocol. The low-voltage activated (LVA) current component contribution was determined by the subtraction method (Bean, 1985): High-voltage activated (HVA) currents obtained at $\mathrm{V}_{H}=-50 \mathrm{mV}$ were subtracted from those obtained at $\mathrm{V}_{H}=-90 \mathrm{mV}$ (HVA plus LVA currents).

\section{Steady-State Analysis}

Steady-state activation (SSA) was analyzed with protocols stepping from $\mathrm{V}_{H}=-90$ (or -50$) \mathrm{mV}$ to $\mathrm{V}_{T}=-90$ to $0 \mathrm{mV}$ $(\Delta \mathrm{V}=10 \mathrm{mV})$ for $12 \mathrm{~ms}$ followed by repolarization to -100 $\mathrm{mV}$ to evoke inward tail currents. Data were fitted by a single Boltzmann function of the form $I_{\max } /\left[1+\exp \left(V_{50}-V\right) / k\right]+m$, were $I_{\max }$ is maximal current, $V_{50}$ is half-voltage of activation, $k$ is slope factor, and $m$ is baseline. Steady-state inactivation (SSI) was determined by stepping the membrane potential to various pre-pulse voltage levels $\left(\mathrm{V}_{\text {pre }}=-110\right.$ to $\left.0 \mathrm{mV}, \Delta \mathrm{V}=10 \mathrm{mV}\right)$ for $1 \mathrm{~s}$ before depolarization to a fixed test level $(-30 \mathrm{mV})$ to evoke channel opening. The resulting data were also fitted to a Boltzmann function.

Steady-state current $\left(\mathrm{I}_{\text {stst }}\right)$ was calculated from the formula $\mathrm{I}=\mathrm{G}^{*}\left(\mathrm{~V}-\mathrm{E}_{\text {Nernst(ion) }}\right)$, where the channel conductance $\mathrm{G}$ is multiplied by the channel's open probability (I/I max, $\mathrm{SSA}$ ) and availability (I/I $\left.\mathrm{I}_{\max }, \mathrm{SSI}\right)$, which were obtained experimentally from the T-type steady state activation (SSA) or inactivation (SSI) curves adjusted to a Boltzmann equation (Bijlenga et al., 2000; Lambert et al., 2014; Rivero-Echeto et al., 2020). Specifically, steady-state current $\left(\mathrm{I}_{\text {stst }}\right)$ was calculated with the steady stated formula Iss $\left(\mathrm{V}_{T}\right)=\mathrm{G} * \mathrm{I} / \mathrm{I}_{\max , S S A}\left(\mathrm{~V}_{T}\right) * \mathrm{I} / \mathrm{I}_{\max , S S I}\left(\mathrm{~V}_{T}\right)^{*}\left(\mathrm{~V}_{T^{-}} 50\right.$ $\mathrm{mV}$ ), where $V_{T}$ is the test voltage, $G$ is the channel conductance (arbitrarily set at 1), I/I $\mathrm{I}_{\max , S S A}$ is the fraction of T-type channels activated at $\mathrm{V}_{T}, \mathrm{I} / \mathrm{I}_{\max }$,SSI is the fraction of T-type channels available at $\mathrm{V}_{T}$ and $50 \mathrm{mV}$ is the experimentally equilibrium potential for calcium.

\section{Resting Membrane Potential}

RMP was recorded in continuous trace mode without current injection for $20 \mathrm{~s}$, and averaged; voltages were corrected for liquid junction potentials.

\section{Input Resistance}

Whole cell input resistance $\left(R_{\text {in }}\right)$ was determined in response to current steps ( -80 to $20 \mathrm{pA}$, stepping each $20 \mathrm{pA}$ ). A holding current was applied to set the membrane potential at $-60 \mathrm{mV}$. 


\section{Membrane Excitability}

AP discharges were triggered by consecutive depolarizing ramps at 20,40 , and $60 \mathrm{pA} / \mathrm{s}$ rates for $1.5 \mathrm{~s}$ (Balasubramanyan et al., 2006; Lu et al., 2006). A holding current was applied to set the membrane potential at $-75 \mathrm{mV}$. The rheobase was determined as the minimum amount of current required for firing an action potential.

\section{Statistical Analysis}

These experiments were exploratory with no pre-determined end-point and no data was excluded from the analyses. No blinding, no randomization, and no sample size calculation were performed. All measurements were done in at least three independent cell cultures. Results are presented as mean \pm SEM. The parameter " $\mathrm{n}$ " indicates number of cells unless otherwise stated and "N" the number of animals. No test for outliers has been applied. The normality of data distribution was assessed by the Kolmogorov-Smirnov test. Statistical analysis was performed with the Sigma Plot 11 Software. Statistical significance $(P<0.05)$ was determined using either one-way analysis of variance (ANOVA) with Fisher LSD's post hoc test for comparisons between multiple groups or an independentsamples $t$-test for comparisons between two groups. Statistical significance $(P<0.05)$ for two proportions was determined using the $Z$-test.

\section{RESULTS}

Hypothalamic cultures were studied from 8 to 11 days in vitro (DIV). Immunocytochemistry analysis (ICC) showed that POMC and NPY/AGRP neurons were present in the culture, with $\mathrm{POMC}^{+}$neurons (POMC) being the majority (85 vs. 15\%, $P<0.05, Z$-test) (Figures 1A,B); note that Figure 1A figure does not display an average distribution of $\mathrm{POMC}^{+}$and $\mathrm{AGRP}^{+}$positive neurons, as it was difficult to find such ratios within the same area during confocal image acquisition. Electrophysiological experiments confirmed $15 \%$ of all studied neurons where neither activated nor inhibited by leptin; whereas $80 \%$ of all neurons were leptin-activated (Figures 1C,D), consistent with the ICC data. In contrast, leptin treatment inhibited $5 \%$ of cells, consistent with an NPY phenotype.

The properties of leptin-activated neurons were consistent with POMC neuron responses. As seen in Figure 2A, their resting membrane potential (RMP) was slightly depolarized, whereas their input resistance remained unaltered upon application of $100 \mathrm{nM}$ leptin (Figure 2B). We assessed the effect of leptin on excitability. Membrane excitability was quantified using depolarizing current ramps $(20,40$, and $60 \mathrm{pA} / \mathrm{s})$ from a preset membrane potential of $\sim-75 \mathrm{mV}$ to avoid spontaneous tonic firing. To analyze quantitatively the voltage response to the current ramps, three different measurements were calculated: the rheobase, the number of APs triggered, and the rate of membrane potential depolarization preceding the first AP (i.e., the slope of depolarization, $\Delta \mathrm{V} / \Delta \mathrm{t}$ ). Figure $2 \mathrm{C}$ shows an example of neuronal firing evoked in a POMC neuron at ramp rates of $20 \mathrm{pA} / \mathrm{s}$. Leptin treatment on its own decreased the rheobase from 18.4 to $14.1 \mathrm{pA}$, increased the number of action potentials from 6 to 11 and enhanced the rate of membrane potential depolarization from 30.0 to $65.0 \mathrm{mV} / \mathrm{s}$; according to the leptinmediated depolarization of the RMP that was observed in Figure 2A. The summary of effects of leptin on neurons is depicted in Figure 2D. On average, for all tested current ramp rates, leptin decreased the rheobase by $\sim 25 \%$ and increased the rate of membrane potential depolarization between $\sim 60$ and $100 \%$. However, leptin increased the AP number by $\sim 64 \%$ only at ramp rates of $20 \mathrm{pA} / \mathrm{s}$.

\section{T-Type Calcium Currents Are Necessary for the Leptin-Induced Excitability Response}

Due to their ability to conduct calcium across the cellular membrane at potentials close to the resting potential, T-type calcium channels are critically important for regulating neuronal excitability (Llinás, 1988; Huguenard, 1996; Perez-Reyes, 2003). Therefore, we were interested in studying the role of T-type calcium currents in the leptin-induced excitability response.

First, we confirmed the presence of $\mathrm{Ca}_{V} 3.1$ and $\mathrm{Ca}_{V} 3.2$ channels in $\mathrm{POMC}^{+}$neurons by immunocytochemistry (Figure 3A) and characterized the calcium currents present in our cultures, including T-type currents (low-voltage gated, LVA). Analysis of $\mathrm{Ca}^{2+}$ currents properties revealed three distinct neuron groups, as seen in the I-V curves depicted in Figure 3B. Of all neurons sampled ( $n=28), 14.3 \%$ expressed only highvoltage activated (HVA) currents; $46.4 \%$ of neurons expressed both HVA and LVA currents at low levels (LD, $\mathrm{I}_{\text {LVApeak }}<6$ -pA/pF), and 39.3\% expressed low HVA and high LVA current density levels (HD, $\mathrm{I}_{\text {LVApeak }}$ from 6 to $20-\mathrm{pA} / \mathrm{pF}$ ) (Figures 3B,C). Currents elicited by a depolarizing pulse from -90 to $-30 \mathrm{mV}$ were confirmed to be T-type by their sensitivity to their specific channel blocker NNC 55-0396 (10 $\mu \mathrm{M}$, Figure 3D).

We next assessed whether T-type channels are part of the cellular pathway of neurons depolarized by leptin. First, we confirmed the presence of TRPC channels in $\mathrm{POMC}^{+}$neurons in our cultures using immunocytochemistry and western blot analysis (Figures 4A, 6). Membrane excitability in control and leptin-treated neurons was studied before and after the application of the TRPC channel blocker 2-APB $(100 \mu \mathrm{M})$ or NNC-55-0396 (10 $\mu \mathrm{M})$ using a ramp rate of $20 \mathrm{pA} / \mathrm{s}$. Figure 4B shows representative examples of leptin-treated neurons before and after aforesaid treatments. In control neurons, neither the application of 2-APB nor the addition of NNC-55-0396 affected neuronal membrane excitability per se (Figure 4C, control, $P>0.05$, ANOVA). However, in leptin-treated neurons, the effect of leptin was abolished after the addition of $2-\mathrm{APB}$, resulting in a $24.5 \pm 4.3 \%$ increase in the rheobase and $48.0 \pm 5.3 \%$ decrease in the number of spikes, compared to the leptin-treated group, corroborating the role of TRPC channels in the leptin-signaling cascade (Figure 4C). Interestingly, the effect of leptin was also prevented solely by the application of the T-type channel blocker NNC-55-0396 (Figure 4C, $P<0.05$, ANOVA). Similarly, both 2-APB and NNC-55-0396 prevented leptin-induced increase 

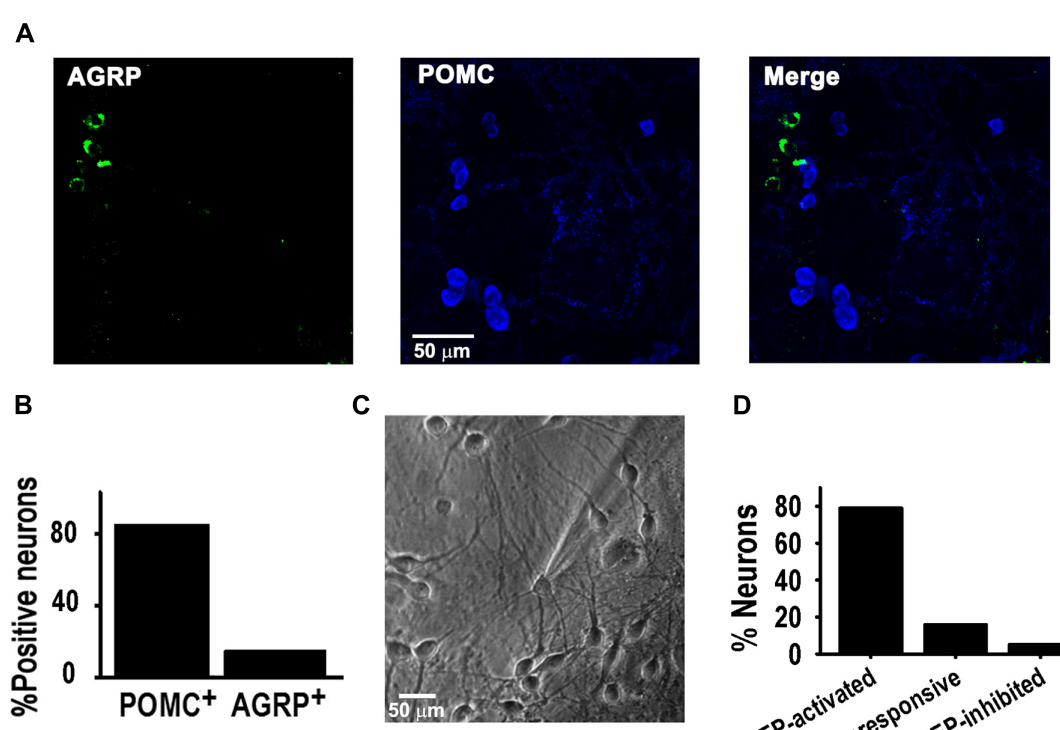

D

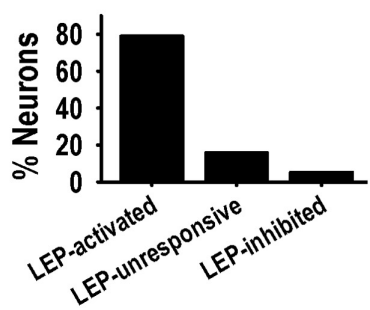

FIGURE 1 | POMC neurons activated by leptin in culture. (A) Example of confocal images showing neurons positive for AGRP (green) or POMC (blue) antibodies in hypothalamus culture at 8-10 DIV. (B) Percentage of neurons positive for AGRP or POMC antibodies $(n=182)$. (C) Microscopic image of cultured hypothalamic neuron at 8 DIV. (D) Percentage of WT neurons activated or inhibited by $100 \mathrm{nM}$ leptin $(n=19)$, using a $20 \mathrm{pA} / \mathrm{s}$ depolarizing ramp stimulus.

of the rate of membrane potential depolarization (Figure 4D, $P<0.05$, ANOVA).

Given that leptin is known to exert direct effects on voltagegated calcium currents (Takekoshi et al., 2001; Wang et al., 2008), we assessed whether acute incubation with leptin altered T-type LVA currents. As seen in Figure 5A, application of 100 $\mathrm{nM}$ leptin in the recording chamber for $30 \mathrm{~min}$ did not alter the T-type peak current density $\left(P>0.05, t\right.$-test, at $\mathrm{V}_{T}=-30$ $\mathrm{mV})$. Furthermore, leptin did not affect either LVA or HVA current-voltage relationships (I-V curve, $P>0.05$, Two Way RM ANOVA, Figure 5B) or the T-type properties of activation or inactivation (not shown), suggesting that the leptin-mediated effect on T-type channels is indirect, likely downstream of TRPC.

So far, our results suggest that T-type calcium currents are as necessary for the leptin-induced excitability response as TRPCs channels. Despite having no direct effect on T-type current density, leptin induced a faster rate of membrane potential depolarization compared to control (Figure 2D); which was prevented after blocking TRPC or T-type channels (Figure 4D). Faster rates of membrane depolarization induce less T-type current inactivation prior to activation of an action potential (Gutierrez et al., 2001), in fact, the results from Figures 4C,D suggest the membrane depolarization rate induced by leptin in response to current ramps of $20 \mathrm{pA} / \mathrm{s}$ favors T-type channel recruitment; in contrast, control neurons exhibited slower membrane potential depolarization rates $(30.3 \pm 2.7$ vs. $66.1 \pm 4.2 \mathrm{mV} / \mathrm{s})$. The latter conditions favor T-type current inactivation, rendering an inadequate number of available $\mathrm{T}$ channels to contribute to the excitability process. Note that NNC-55-0396 did not affect the excitability in control neurons (Figures 4C,D). Therefore, we hypothesize that increased T-type channel availability resulting from the faster leptin-induced membrane potential depolarization rate is sufficient to increase membrane excitability and trigger sodium-mediated action potentials in POMC neurons.

In order to evaluate our hypothesis, we first studied whether T-type channels could be recruited by rates of membrane potential depolarization faster than $30 \mathrm{mV} / \mathrm{s}$ in control neurons. We assessed the T-type current activation in voltage-clamp configuration in response to increasing rates of membrane potential depolarization preceding the first AP (left panel, Figure 5C). Peak currents for each rate of membrane potential depolarization were normalized to the maximally elicited current. Average values were plotted against its respective rate of membrane potential depolarization (slope) and fitted using a Boltzmann equation (right panel, Figure 5C). The approximate rate of membrane potential depolarization in response to a ramp rate of $20 \mathrm{pA} / \mathrm{s}$ for control ( $\sim 30 \mathrm{mV} / \mathrm{s}$, black lines) and leptintreated ( $\sim 70 \mathrm{mV} / \mathrm{s}$, red lines) neurons are shown on the voltage axis of Figure $5 \mathrm{C}$. The lines interpolating the $Y$-axis denote the percentage of non-inactivated $\mathrm{I}_{T}$ that is recruited in response to the respective rate of membrane potential depolarization. In control neurons (black line), only around $\sim 5 \%$ of $\mathrm{I}_{T}$ is available for recruitment by a membrane depolarization rate of $\sim 30 \mathrm{mV} / \mathrm{s}$. However, membrane depolarization rates similar to those induced by leptin-treatment $(\sim 70 \mathrm{mV} / \mathrm{s})$ would be able to recruit $20 \%$ of $\mathrm{I}_{T}$. Thus, control neurons were stimulated in current-clamp configuration with depolarizing current ramps of $40 \mathrm{pA} / \mathrm{s}$, which produce on average a membrane depolarization rate of $73.8 \pm 9.1 \mathrm{mV} / \mathrm{s}$. As seen in Figure 5D, NNC-550396 not only caused a large increase in the rheobase, but also significantly decreased the number of AP in control neurons. 

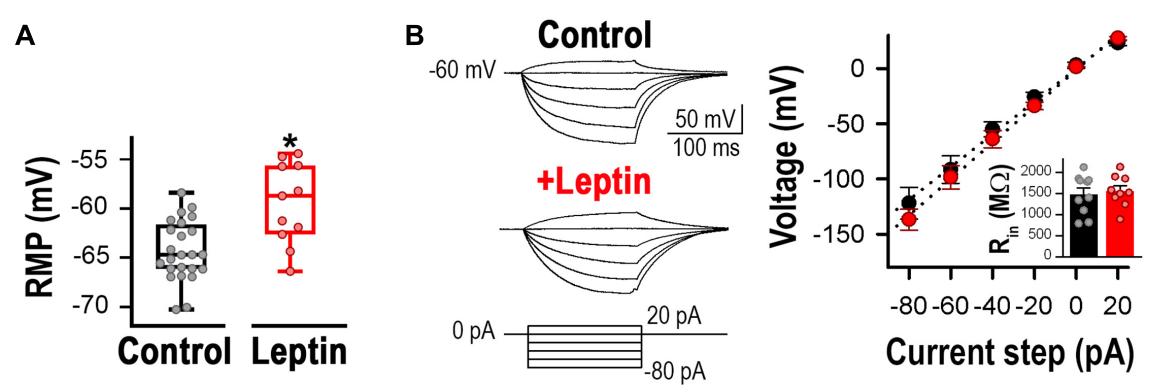

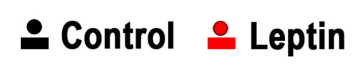

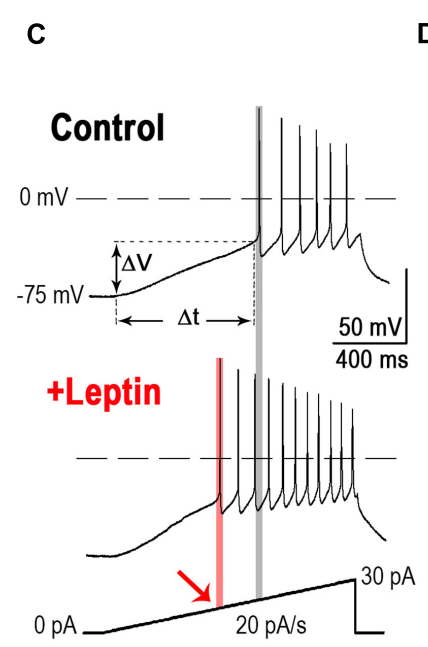

D
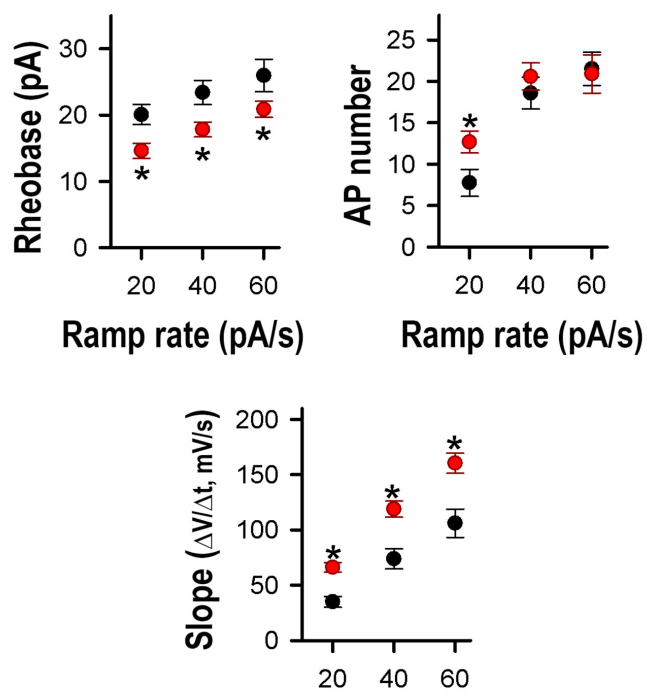

Ramp rate $(\mathrm{pA} / \mathrm{s})$

FIGURE 2 | Leptin increases excitability in cultured hypothalamic neurons. (A) Leptin application induced a $\sim 6 \mathrm{mV}$ depolarization. Resting membrane potential (RMP) from control $(n=24)$ and leptin-treated $(n=11)$ neurons. * Significantly different from control, $P<0.05, t$-test $(t=3.923$, df $=33)$. (B) Input resistance $\left(R_{i n}\right)$ from control $(n=10)$ and leptin-treated neurons $(n=9)$. Representative voltage traces (left) and average voltage-current ( $\mathrm{V}$-I) relation (right) in response to $200 \mathrm{~ms}$ current steps from -80 to $20 \mathrm{pA}$ (every $20 \mathrm{pA}$, preset membrane potential $=-60 \mathrm{mV}$ ). $R_{\text {in }}$ was calculated as the slope of the linear regression. No changes in $R_{\text {in }}$ were observed between control and leptin treatments $(P>0.05, t$-test, $t=0.3883$, $\mathrm{df}=17)$. (C) Examples of neuronal firing in control and leptin-treated neurons elicited from a preset membrane potential of $-75 \mathrm{mV}$ by a $20 \mathrm{pA} / \mathrm{s}$ ramp. The rheobase is indicated with the gray line in control and the pink line and arrow in leptin-treated neurons. (D) Quantification of rheobase (pA), number of action potentials, and rate of membrane potential depolarization (Slope $=\Delta \mathrm{V} / \Delta \mathrm{t}$, $\mathrm{mV} / \mathrm{s}$, see panel C) in control $(n=24,24,12)$ and leptin-treated $(100 \mathrm{nM}, n=19,19,15)$ neurons in response to 20,40 and $60 \mathrm{pA} / \mathrm{s}$ ramps. *Significantly different from control, $P<0.05$, $t$-test. Rheobase: $t=2.744$ for $20 \mathrm{pA} / \mathrm{s}, t=2.565$ for $40 \mathrm{pA} / \mathrm{s}, t=1.718$ for $60 \mathrm{pA} / \mathrm{s}$, df $=41$. AP number: $t=2.257$ for $20 \mathrm{pA} / \mathrm{s}, t=0.766$ for $40 \mathrm{pA} / \mathrm{s}$, $t=0.198, \mathrm{df}=41$. Slope: $t=4.030$ for $20 \mathrm{pA} / \mathrm{s}, t=2.908$ for $40 \mathrm{pA} / \mathrm{s}, t=2.894$ for $60 \mathrm{pA} / \mathrm{s}, \mathrm{df}=25$.

These results confirm that fast rates of membrane depolarization in response to excitatory stimuli recruit enough non-inactivated T-type channels to contribute to the membrane excitability.

Overall, our results show that T-type channel recruitment is necessary in leptin-mediated pathway, likely downstream of TRPC channel activity, as summarized in the cartoon in Figure 5E.

\section{T-type Calcium and TRPC1/5 Channels Are Expressed in a Functional Complex}

We explored whether TRPC and T-type channels exist in a complex. TRPC1/5 complexes are likely the physiological mediators of leptin's effects in the hypothalamus
(Strübing et al., 2001), therefore we assessed whether $\mathrm{Ca}_{V} 3$ channels co-exist with TRPC5 channels in mouse whole brain and hypothalamus samples. Figure $\mathbf{6 A}$ shows that $\mathrm{Ca}_{V} 3.1$ channels co-precipitate with TRPC5 channels (left), and similarly, TRPC5 channels co-precipitate with $\mathrm{Ca}_{V} 3.1$ channels (right panel). $\mathrm{Ca}_{V} 3.2$ channels also co-precipitate with TRPC5 channels (Figure 6B, left), and TRPC5 interact with $\mathrm{Ca}_{V} 3.2$ (right). We also determined that TRPC1 is detected in samples co-precipitated with TRPC5 and $\mathrm{Ca}_{V} 3.1$ (left) or $\mathrm{Ca}_{V} 3.2$ (right) (Figure 6C), confirming a TRPC multimer formed by TRPC1/5 is present in the hypothalamus.

Because T-type calcium channels co-exist in a complex with TRPC1/5, it is possible that local membrane depolarization induced by TRPC1/5 channel-mediated cation influx recruits 
A
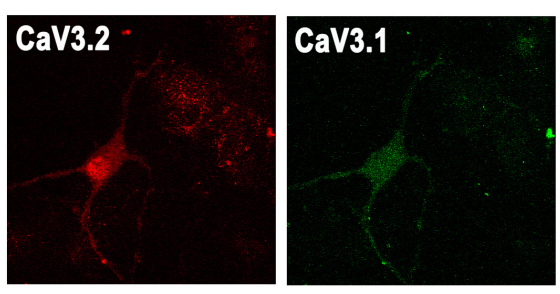

C

B

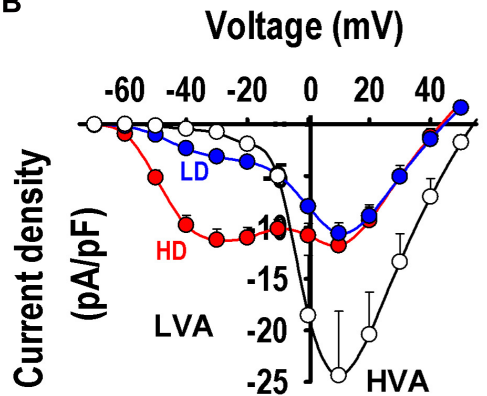

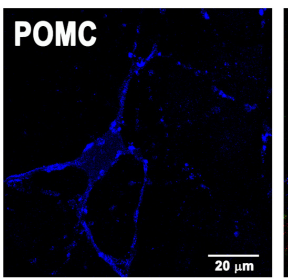

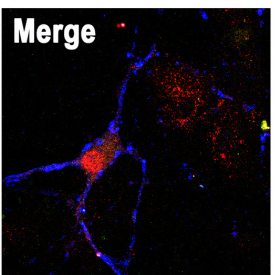

D
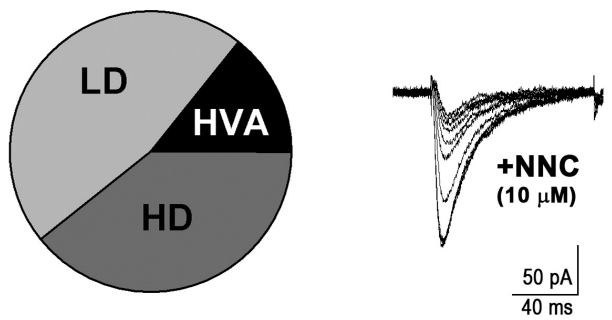

FIGURE 3 | T-type calcium channels in POMC neurons. (A) Example of fluorescence images showing the expression of T-type $\alpha_{1 H}\left(\mathrm{Ca}{ }_{V} 3.2\right.$, red) and $\alpha_{1 G}\left(\mathrm{Ca}_{V} 3.1\right.$, green) channels in a POMC neuron (blue). Bar size, $20 \mu \mathrm{m}$. (B) I-V curves depict three distinct neuron groups ( $n=28)$ : empty circles, neurons expressing only high-voltage activated (HVA) currents; blue circles, neurons expressing low HVA and low LVA current density levels (LD, ILVApeak < 6 -pA/pF); and red circles, neurons expressing low HVA and high LVA current density (HD, ILVApeak from 6 to 20 -pA/pF). (C) Pie chart denotes the percentual number of neurons in each group described in (A). (D) T-type calcium current blockade by its specific blocker NNC 55-0396 (10 $\mu \mathrm{M})$; the trace amplitude decrement sequence is shown every 2 min before (larger trace) and during application of the drug.

T-type channel activity. As the ionic selectivity for TRPC1/5 channel complexes is almost $1: 1$ for $\mathrm{Na}^{+}$and $\mathrm{Ca}^{2+}, \mathrm{Ca}^{2+}$ ions contribute two thirds of the total TRPC1/5-mediated depolarization. Accordingly, chelation of $\mathrm{Ca}^{2+}$ influx with a fast chelator that binds $\mathrm{Ca}^{2+}$ closer to the mouth's pore (BAPTA$\left.\mathrm{AM}, \mathrm{K}_{O N}=6 \times 10^{8}\right)$ compared to a slower, global chelator $\left(\right.$ EGTA-AM, $\left.\mathrm{K}_{O N}=1.5 \times 10^{6}\right)$ should allow us to discern whether the TRPC1/5 and T-type channels complex function within a microdomain (Figure 7A). We incubated cultured hypothalamic neurons with either $10 \mu \mathrm{M}$ BAPTA-AM or EGTA$\mathrm{AM}$ for $30 \mathrm{~min}$ at $37^{\circ} \mathrm{C}$ prior to whole-cell current clamp to assess excitability. As expected, leptin depolarized the RMP via activation of TRPC channels (Figure 7B, $P<0.05$, ANOVA; RMP values for control and leptin-treated neurons have been reproduced from Figure $\mathbf{2 A}$ for comparison). The depolarizing effect of leptin occurred regardless of incubation with either BAPTA or EGTA (Figure 7B, $P<0.05, t$-test), suggesting that the chelation of calcium does not affect the TRPC1/5 function per se. Figure 7C shows example traces of APs elicited with a $20 \mathrm{pA} / \mathrm{s}$ ramp protocol under control conditions (top trace), and after the addition of $100 \mathrm{nM}$ leptin in the presence of BAPTA (middle) or EGTA (bottom). As expected, the presence of intracellular BAPTA or EGTA did not alter the baseline excitability response compared to control $(P>0.05$, ANOVA; the rheobase and AP number values for control and leptin-treated neurons have been reproduced in Figure 7D from Figure 4 for comparison). Addition of $100 \mathrm{nM}$ leptin in the presence of EGTA caused the expected increase in excitability $(P<0.05, t$-test $)$, in contrast with leptin in the presence of internal BAPTA, which did not elicit significant changes in either the number of APs, rheobase or rate of membrane potential depolarization $(P>0.05, t$-test). Our results suggest that calcium mediates leptin effects on membrane excitability in the proximity of the leptin effector, supporting the idea that TRPC1/5 and T-type channels complex could function within a microdomain.

To further confirm our results, T-type channels were recruited by using depolarizing ramps of $40 \mathrm{pA} / \mathrm{s}$. Neither BAPTA nor EGTA affected membrane excitability in control neurons under those conditions, suggesting no direct effects on the contribution of T-type channels to basal neuronal excitability (Figure 7E). However, only BAPTA prevented leptin-mediated effects on membrane excitability (Figure 7E) in a similar manner as observed with $20 \mathrm{pA} / \mathrm{s}$ depolarizing ramps (Figure 7D). These results indicate that the BAPTA effects on membrane excitability were restricted to local leptin-mediated recruitment of T-type channels. The lack of effect of EGTA on membrane excitability confirms that leptin does not depend on a global calciumdependent mechanism to regulate excitability.

Overall, these results support the hypothesis that $\mathrm{Ca}^{2+}$ influx via TRPC1/5 and subsequent depolarization enhances the recruitment of $\mathrm{Ca}_{V} 3$ channels, favoring the generation of APs.

We further studied the physiological relevance of this TRPC1/5-CaV3 complex. As seen in Figures $\mathbf{2 A}, \mathbf{7 B}$, the application of leptin induced a $\sim 6 \mathrm{mV}$ depolarization of RMP. Figure 7F (top panel) shows the steady-state activation (SSA) and steady-state inactivation (SSI) properties of T-type current 
A
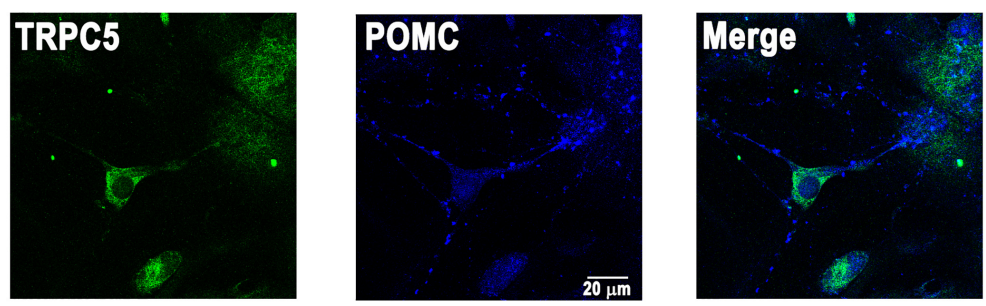

B

C
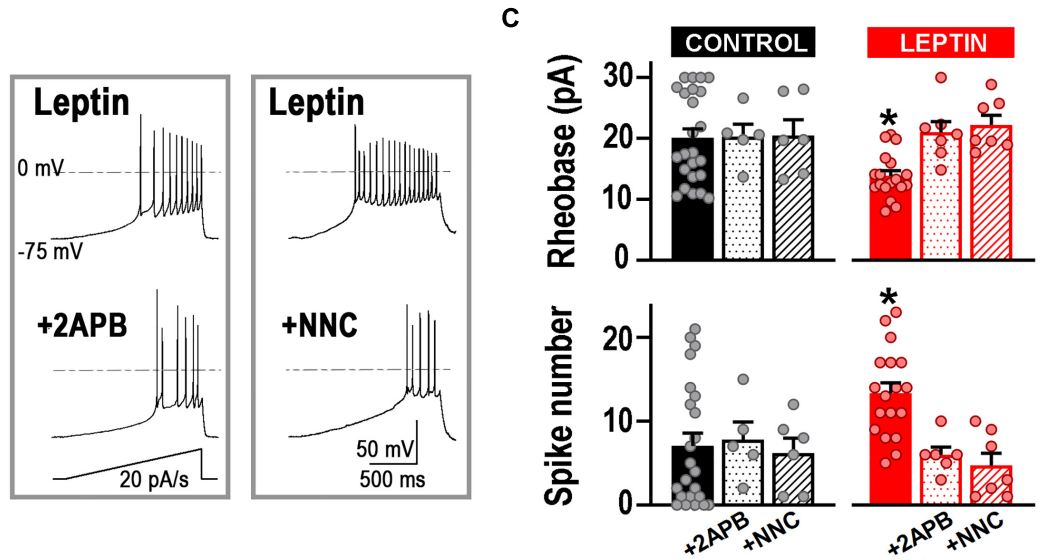

D

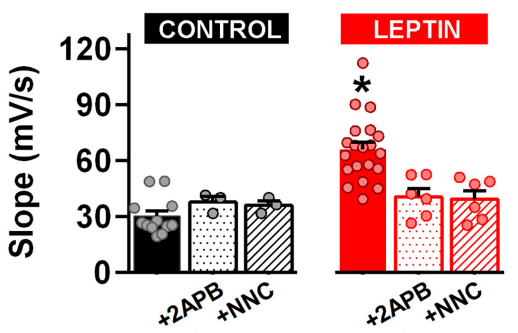

FIGURE 4 | T-type calcium channels are necessary for the leptin-induced excitability response. (A) Example of fluorescence images showing the expression of TRPC5 channels (green) in POMC neurons (blue). (B) Examples of neuronal firing elicited from a preset membrane potential of $-75 \mathrm{mV}$ by a $20 \mathrm{pA} / \mathrm{s}$ ramp in leptin-treated neurons before and after the addition of the TRPC channel blocker 2-APB (100 $\mu \mathrm{M})$ or the T-type channel blocker NNC 55-0396 (10 $\mu \mathrm{M})$. (C) Quantification of rheobase $(\mathrm{pA})$ and number of action potentials in control neurons $(n=24)$, neurons treated with the TRPC channel blocker 2-APB (100 $\mu$ M, $n=5)$, neurons treated with the T-type channel blocker NNC 55-0396 (10 $\mu \mathrm{M}, n=6)$, neurons treated with leptin $(100 \mathrm{nM}, \mathrm{n}=18)$, neurons treated with leptin and 2-APB $(n=5)$, and neurons treated with leptin and NNC 55-0396 (10 $\mu \mathrm{M}, n=7)$. * Significantly different from all others, $P<0.05$, ANOVA, $F_{(5,61)}=3.786$ (for rheobase), $F_{(5,61)}=3.856$ (for AP number). (D) Quantification of the rate of membrane potential depolarization (slope) in control neurons $(n=12)$, neurons treated with the TRPC channel blocker 2-APB (100 $\mu \mathrm{M}, n=3)$, neurons treated with the T-type channel blocker NNC 55-0396 $(10 \mu \mathrm{M}, n=3)$, neurons treated with leptin (100 nM, $n=20)$, neurons treated with leptin and 2-APB $(n=6)$, and neurons treated with leptin and NNC 55-0396 (10 $\mu \mathrm{M}, n=6)$. *Significantly different from all others, $P<0.05$, ANOVA, $F_{(5,44)}=12.77$.

in cultured hypothalamic neurons. The superimposed lines on the graph show the interpolated values of RMP ( $Y$-axis) for control (black lines) and leptin-treated neurons (red lines) pinpointing the non-inactivated (available, SSI) and the activable [open probability (PO), SSA] fractions of T-type channels at the respective resting membrane potentials. We calculated the T-type window current using the fitting parameters of the steadystate activation and inactivation curves (see section "Material and Methods") (Bijlenga et al., 2000; Lambert et al., 2014; RiveroEcheto et al., 2020), which is shown in turquoise encompassing the region underneath the steady-state curves (Figure 7F, top panel). Of note, T-type channels are hardly functional during control conditions, when the $50 \%$ of available channels are not highly activable.

Our results show that the leptin-mediated RMP depolarization induces changes in T-type channel availability (SSI) and open probability (SSA) (Figure 7F, top panel, red line), leading to a voltage right-shift into the active T-type window current range that results in an increase of the steady state T-type calcium current from 40 to $70 \%$, as better seen in the normalized steady state current graph (Figure $7 \mathbf{F}$, bottom panel). These leptin-mediated changes on T-type calcium steady state current ultimately alter the intrinsic excitability of POMC neurons. 


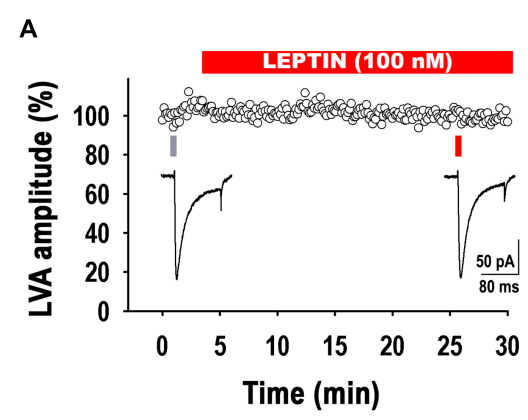

C

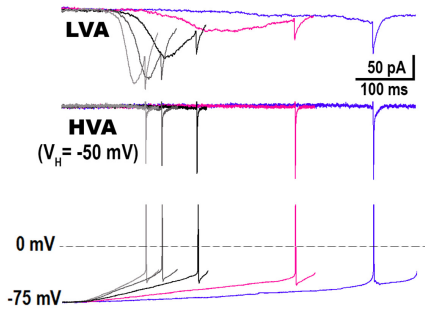

D

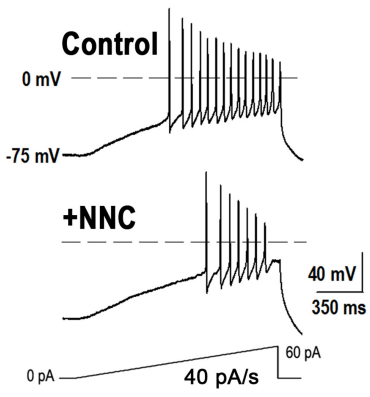

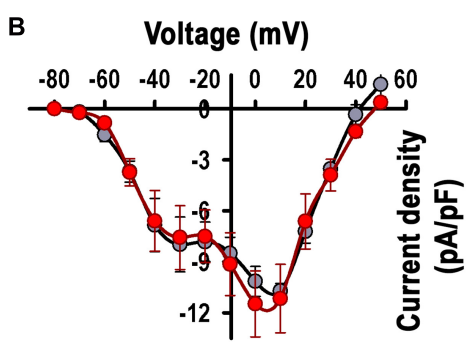

$\multimap-$ Control $\longrightarrow+$ Leptin

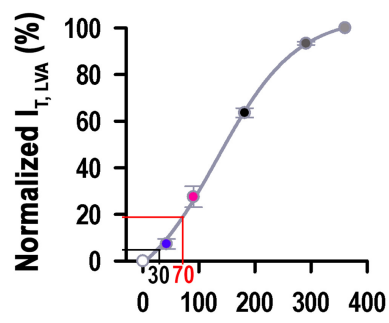

Slope $(\mathrm{mV} / \mathrm{s})$

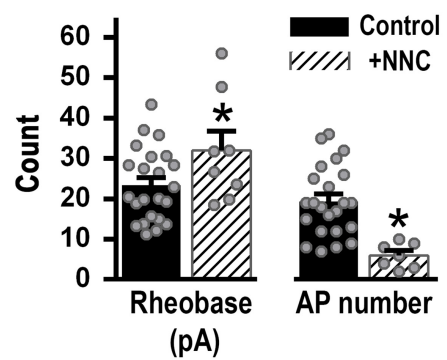

E

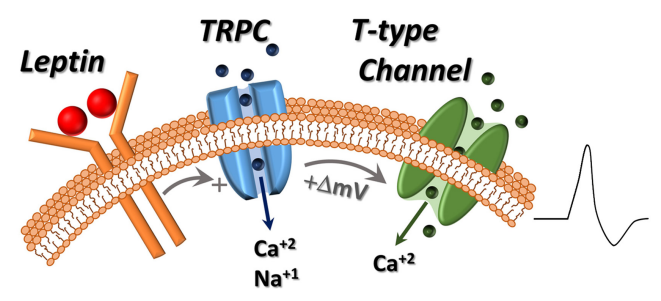

FIGURE 5 | Leptin has no direct effect on T-type current density but increases the membrane depolarization rate favoring T-type channels recruitment. (A) Acute incubation with Lep (100 nM) does not alter T-type currents (i.e., LVA currents) elicited by a step voltage from -90 to $-30 \mathrm{mV}(n=3)$. (B) I-V curves before and after the application of $100 \mathrm{nM}$ leptin in the recording chamber $\left(n=8\right.$, paired experiments). Currents were elicited by depolarizing voltage steps $(\Delta \mathrm{V}=10 \mathrm{mV})$ from a $\mathrm{V}_{H}$ of -90 to $50 \mathrm{mV}$. There is not a statistically significant difference before and after the addition of leptin $\left[P>0.05\right.$, Two Way RM ANOVA, $\left.F_{(1,55)}=0.221\right]$. There is not a statistically significant interaction between the voltage and the treatment $\left[P>0.05\right.$, Two Way RM ANOVA, $\left.\left.F_{(11}, 55\right)=0.785\right]$. I-V curves from neurons that expressed LVA current at low and high levels were pooled. (C) Left: Average traces of T-type currents elicited by various rates of voltage depolarization preceding the first AP. Stimulation protocols (bottom traces) were obtained from hypothalamic neurons recorded in current clamp configuration during current ramp stimulations (20-100 $\mathrm{pA} / \mathrm{s})$, which resulted in the following rates of membrane potential depolarization prior the first AP: 40, 90, 180, 290 or $360 \mathrm{mV} / \mathrm{s}\left(\mathrm{V}_{H}=-75 \mathrm{mV}\right)$. Before stimulation, the membrane potential was held at -90 or $-50 \mathrm{mV}\left(\mathrm{V}_{H}\right)$ in order to isolate T-type currents. HVA currents recorded from a $\mathrm{V}_{H}$ of $-50 \mathrm{mV}$ (middle traces) were subtracted from LVA and HVA currents recorded from a $V_{H}$ of $-90 \mathrm{mV}$, obtaining the isolated LVA component (top traces). Right: Normalized T-type current as function of the membrane depolarization rate (slope) $(n=5)$. The black line marks the percentage of non-inactivated T-type current recruited by a depolarizing rate of $30 \mathrm{mV} / \mathrm{s}$. The red line marks the percentage of non-inactivated T-type current recruited by a depolarizing rate of $70 \mathrm{mV} / \mathrm{s}$. (D) Left: Example of neuronal firing elicited from a preset membrane potential of $-75 \mathrm{mV}$ by a $40 \mathrm{pA} / \mathrm{s}$ ramp in a control neuron before and after the addition of the T-type channel blocker NNC $55-0396$ (10 $\mu \mathrm{M})$. Right: Application of NNC 55-0396 $(n=8)$ reduced membrane excitability in comparison to control $(n=23)\left(-75 \mathrm{mV}, 40 \mathrm{pA} / \mathrm{s}\right.$ ramp). ${ }^{*}$ Significantly different from control, $P<0.05, t$-test. Rheobase: $t=2.060, \mathrm{df}=29$. AP number: $t=3.956, \mathrm{df}=29$. (E) Updated model of leptin's cellular signaling. Leptin binding to its receptor results in activation of the PI3 kinase signaling pathway and subsequent activation of TRPC channel. TRPC channel activity induces a small but rapid membrane depolarization that recruits T-type channel activity, increasing hypothalamic neuron excitability. 


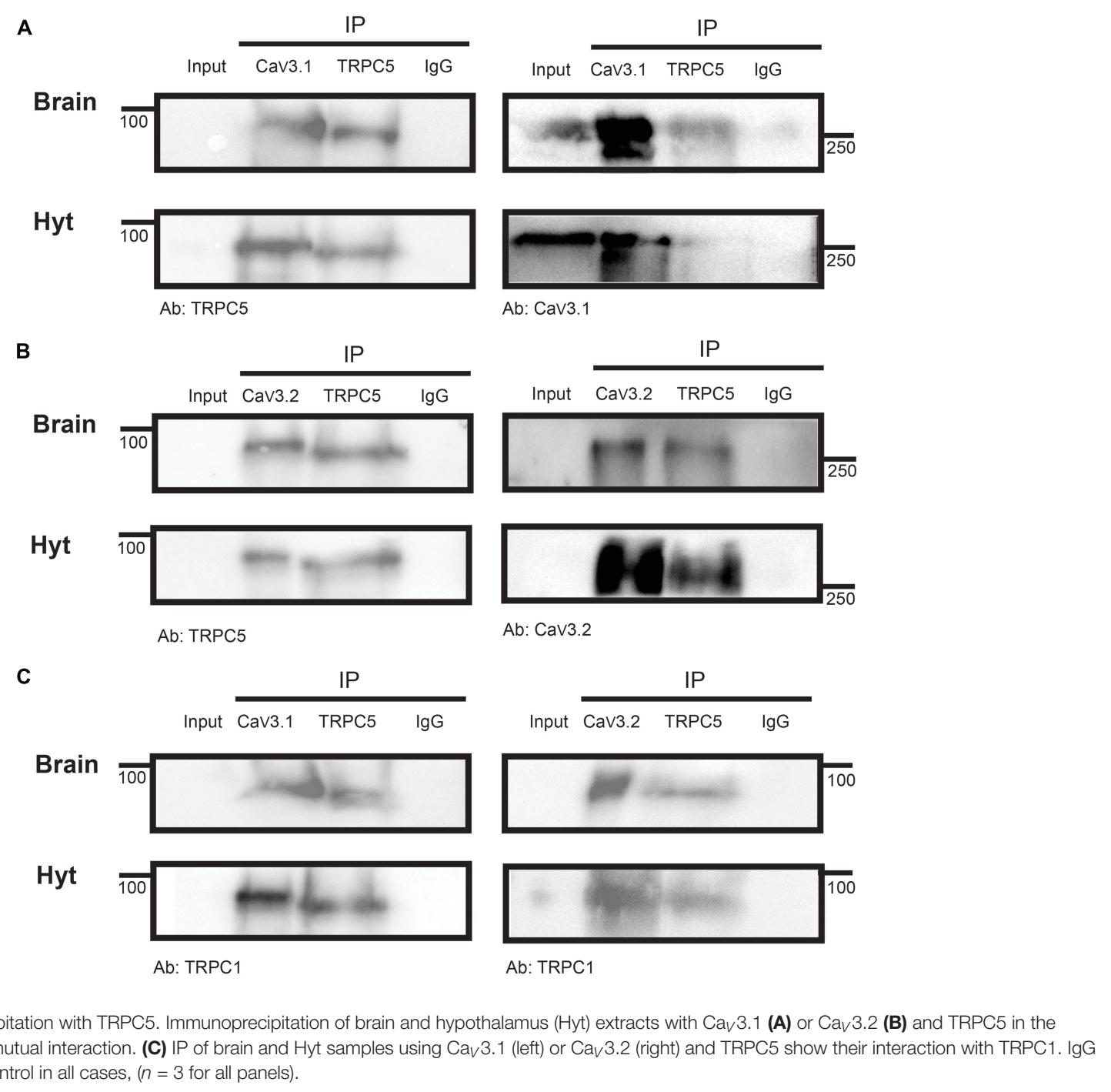

\section{DISCUSSION}

Neuronal cultures with fully developed synapses (after 7 DIV) are useful for mechanistic studies, as they display an "adult" pattern behavior, including ion channel expression (Schlick et al., 2010). Here we corroborated that our primary POMC ${ }^{+}$ hypothalamic neurons responded to leptin as expected according to the bibliography. It is well-established that leptin's effect on POMC neurons is mediated by the activation of TRPC channels (Qiu et al., 2010, 2011, 2014), POMC neurons increased in our system their excitability in response to administration of nanomolar levels of leptin and this effect was mediated through the activation of TRPC channels, in line with the literature (Qiu et al., 2010, 2011, 2014). The present work provides additional detail on this mechanism, demonstrating that TRPC and T-type channels coexist in a physical and functional macromolecular complex, whereby, leptin-induced membrane depolarization via TRPC channel activity immediately recruits adjacent T-type channels, which further depolarize POMC neurons triggering an increase in intrinsic excitability.

Hyperpolarization-induced removal of T-type channel inactivation allows for their stimulation by small depolarizations near the resting potential, rendering T-type currents optimal for regulating excitability under physiological conditions near resting state; as such, T-type channels help control neuronal excitability in various hypothalamic nuclei (van den Top et al., 2004; Qiu et al., 2006; Bosch et al., 2009; Zhang et al., 2009). T-type channels have a confirmed role linking thalamocortical central regulation of wakefulness and body weight (Uebele et al., 2009), and they have been proposed as potential therapeutic targets for treating obesity (Chemin et al., 2001; Uebele et al., 2009; Chorvat, 2013). T-type antagonists prescribed for epilepsy, depression, obsessive-compulsive disorder and bulimia nervosa can cause loss of appetite as a side effect (Cookson and Duffett, 1998; Traboulsie et al., 2006; Wilfong and Willmore, 2006). Here we establish T-type channels $\left(\mathrm{Ca}_{V} 3\right)$ as essential mediators of membrane depolarization and neuronal excitability triggered 
A

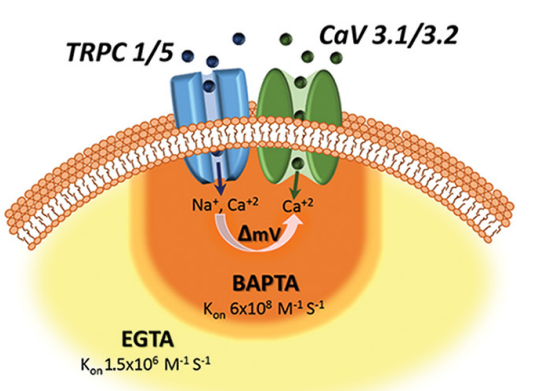

C

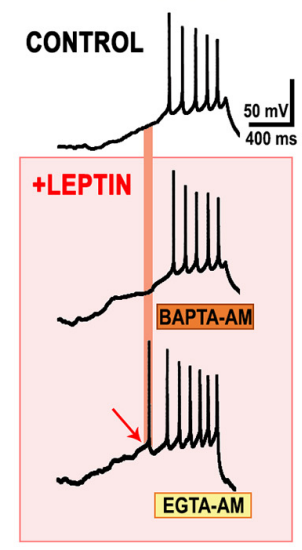

$\mathrm{E}$

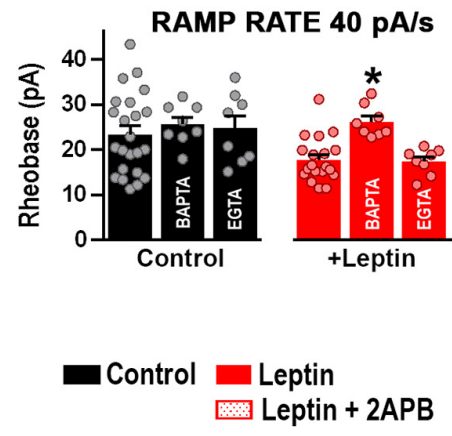

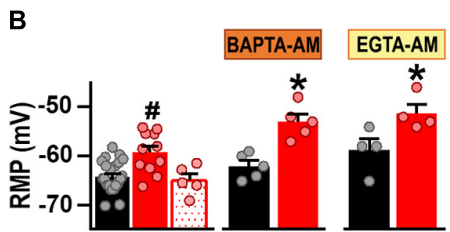

D RAMP RATE $20 \mathrm{pA} / \mathrm{s}$
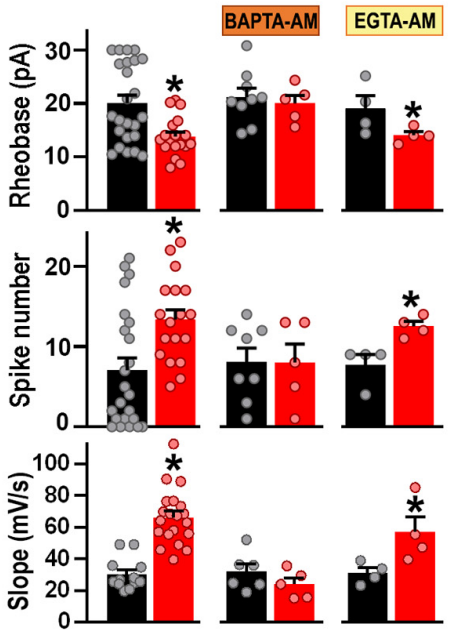

$\mathbf{F}$
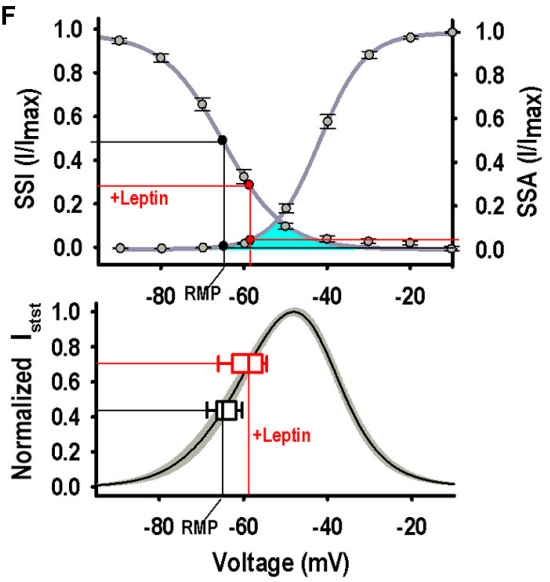

FIGURE $7 \mid \mathrm{Ca}^{2+}$ influx via TRPC1/5 depolarizes and recruits T-type channels. (A) Cartoon depicting T-type calcium channels co-exist in a complex with TRPC1/5. Membrane depolarization induced by TRPC channel-mediated cation influx recruits T-type channel activity. Chelation of $\mathrm{Ca}^{2+}$ influx with a fast chelator that binds $\mathrm{Ca}^{2+}$ closer to the mouth's pore (BAPTA-AM, $\left.\mathrm{K}_{\mathrm{ON}}=6 \times 10^{8}\right)$ is represented in orange. Chelation of $\mathrm{Ca}^{2+}$ influx with a slower chelator $\left(\mathrm{EGTA}-\mathrm{AM}, \mathrm{KON}=1.5 \times 10^{6}\right.$ ) is represented in light orange. (B) Resting membrane potential (RMP) from control $(n=24)$ and leptin-treated neurons in the absence $(n=11)$ or presence of 2APB $(n=5)$. Values for control and leptin-treated neurons were reproduced from (A) for comparison. Cultured hypothalamic neurons were incubated with either $10 \mu \mathrm{M}$ BAPTA-AM $(n=5)$ or EGTA-AM $(n=4)$ for 30 min at $37^{\circ} \mathrm{C}$ prior to whole-cell current clamp to assess leptin-mediated effects on the RMP. " Significantly different from others, $P<0.05$, ANOVA, $F_{(2,37)}=8.927$. ${ }^{*}$ Significantly different from control, $P<0.05$, $t$-test. BAPTA: $t=4.743$, df $=8$. EGTA: $t=2.579$, df $=6$.

(C) Example traces of APs elicited by a $20 \mathrm{pA} / \mathrm{s}$ ramp protocol under control conditions (top trace), and after the addition of $100 \mathrm{nM}$ leptin in the presence of BAPTA (middle) or EGTA (bottom). (D) Rheobase, AP number, and slope values elicited by $20 \mathrm{pA} / \mathrm{s}$ ramps for each treatment $(n=4-24)$. *Significantly different from control, $P<0.05$, $t$-test. Rheobase: $t=3.285$ ( $\mathrm{df}=40$, control), $t=0.4757$ ( $\mathrm{df}=12$, BAPTA incubation), $t=2.019$ ( $\mathrm{df}=6$, EGTA incubation). AP number: $t=3.065$ (df $=39$, control), $t=0.044$ ( $\mathrm{df}=11$, BAPTA), $t=3.376$ ( df =6, EGTA). Slope: $t=6.495$ ( $\mathrm{df}=30$, control), $t=1.245$ (df = 9, BAPTA incubation), $t=2.446$ (df $=6$, EGTA incubation). (E) Rheobase values elicited by $40 \mathrm{pA} / \mathrm{s}$ ramps for each treatment $(n=8-23)$. *Significantly different from others, $P<0.05$, ANOVA, $F(2,36)=0.2637$ for control neurons, $F_{(2,32)}=12.36$ for leptin-treated neurons. (F) T-type channel availability (SSI) and open probability (SSA) as function of voltage. SSI parameters: $V_{50}=-65.4 \pm 1.0 \mathrm{mV}, \mathrm{k}=7.1 \pm 0.5(n=21)$. SSA parameters: $\mathrm{V}_{50}=-41.8 \pm 0.7 \mathrm{mV}, \mathrm{k}=5.2 \pm 0.3(n=22)$. Black circles indicate the availability and the open probability of T-type channels) at the indicated RMP. Red circles indicate steady sate properties at depolarized RMP after leptin (100 nM) incubation. Window current, underneath SSA and SSI curves is indicated in turquoise. Window currents were calculated for each recording neuron from the SSA and SSI curves fitted with the Boltzmann function ( $n=18$, see section "Materials and Methods"); SEM is indicated in gray. The steady-state current was normalized to its maximum value (Normalized Isst). Steady-state current at the RMP (empty black box plot) increased after incubation with leptin (empty red box plot). 
by activation of the JNK2-PI3K cascade in response to leptin in POMC neurons. Although leptin has many functions, including effects on control of hormone release, immune system, vasculature, development, somatosensory thalamic activity and higher-level cognitive functions (Haynes et al., 1999; Kim and Moustaid-Moussa, 2000; Friedman, 2004; Myers et al., 2008; Domingos et al., 2011; Farr et al., 2014; Perissinotti et al., 2018); one of its prominent roles is as effector of the negative feedback loop, supporting homeostatic control of energy and food intake, and adipose tissue mass (Ahima et al., 1996). The majority of neurons in our hypothalamic cultures were leptin-activated, in line with a higher abundance of POMC positive neurons detected by ICC. In these neurons, leptin induces electrical activity and depolarization via binding to LRb, activation of Janus tyrosine kinase 2 and the downstream activation of phosphatidylinositol 3 kinase (PI3K), resulting in activation of transient receptor potential cation channel activity (TRPC) (Harvey, 2007; Qiu et al., 2010, 2011, 2014). Indeed, leptin application to cultured hypothalamic neurons in vitro also resulted in membrane depolarization and increased neuronal excitability. Treatment with the TRPC channel blocker 2-APB after leptin application produced a decrease in the excitability, which was measured as an increase in the rheobase and a decrease of both the rate of membrane depolarization and the number of spikes. However, we found that in addition to TRPC channel activation, T-type channel activity is also essential in this pathway. Blockade of T-type channels with NNC 55-0396 completely prevented the effect of leptin, even when TRPC channel activity remained intact. Qiu et al. (2010) demonstrated leptin recruits a nonselective $\mathrm{Na}^{+}$and $\mathrm{Ca}^{2+}$ channel sensitive to SKF96365 and APB and potentiated by lanthanum $\left(\mathrm{La}^{3+}\right)$, consistent with a TRPC channel complex. Here, we showed T-type channel function is essential in the response to leptin. The leptin-activated current is strongly potentiated by lanthanum (Qiu et al., 2010), a T-type channel blocker more potent than nickel (Mlinar and Enyeart, 1993). Thus, it' is unlikely T-type calcium channels are directly activated by leptin, consistent with our data showing leptin does not directly activate these channels and the steady-state properties of these currents. Instead, leptin induced faster rates of membrane depolarization, which in turn produced less T-type current inactivation prior to activation of an action potential (Gutierrez et al., 2001). Therefore, T-type channels are likely recruited downstream of TRPC channel activation by discrete changes in membrane depolarization induced by TRPC channels and are the final mediator of triggered activity. Insulin and leptin engage a common signaling pathway at the cellular level to activate TRPC5/1 channel complexes and depolarize POMC neurons (Qiu et al., 2014). Interestingly, estradiol-mediated upregulation of Cav3.1 channel rendered POMC neurons more excitable and responsive to insulin-mediated TRPC5 channel activation (Qiu et al., 2018).

Furthermore, we demonstrate TRPC1/C5 channels and $\mathrm{Ca}_{V} 3.1$ and $\mathrm{Ca}_{V} 3.2$ channels exist in complex. Calcium channels are known to co-exist with other channels in functional complexes (Robitaille et al., 1993; Vivas et al., 2017), notably T-type channels form protein complexes with members of the potassium channel family such as $\mathrm{Kv} 4$, KCa3.1, and KCa1.1
(Anderson et al., 2010; Rehak et al., 2013), which ensures rapid potassium channel activation thanks to their proximity with $\mathrm{Ca}_{V} 3$ channels within the microdomain. Furthermore, TRPC channels are known to assemble in multiprotein complexes that include various key $\mathrm{Ca}^{2+}$ signaling proteins within $\mathrm{Ca}^{2+}$ signaling microdomains (Ambudkar, 2006). For instance, TRPC1, 3, 4, 5, 6, and 7 isoforms can form a macromolecular complex with the $\alpha_{1 C}$ subunit of the L-type voltage-gated calcium channel (Cav1.2) in atria and ventricle of developing heart (Sabourin et al., 2011). Here, co-immunoprecipitation experiments show that TRPC5 interacts with $\mathrm{Ca}_{V} 3.1$ and $\mathrm{Ca}_{V} 3.2$ channels. The functional activity of this complex was corroborated using intracellular calcium chelators; prevention of leptin-induced calcium influx through TRPC channels by intracellular BAPTA (but not EGTA) was sufficient to preclude POMC neuron excitability. Our results agree with those of Qiu et al. (2010), who reported that leptin-induced inward current was reduced by BAPTA but not EGTA. It has been shown that intracellular BAPTA prevents intracellular $\mathrm{Ca}^{2+}$-dependent potentiation of the TRPC channel complex in vitro, suggesting that calcium binding to an intracellular site in the TRPC channel complex is necessary for its function (Strübing et al., 2001). However, leptin is capable of recruiting a non-selective $\mathrm{Na} / \mathrm{Ca}$ current in hypothalamic neurons in the presence of BAPTA, albeit smaller than in control conditions but larger than that observed in the absence of extracellular calcium (Qiu et al., 2010). In line with these results, we show that leptin-induced depolarization can occur even in the presence of either BAPTA or EGTA suggesting that the calcium entry thought to be necessary to self-activate the TRPC1/5 complex is not blocked by the presence of BAPTA in hypothalamic neurons.

T-type $\mathrm{Ca}^{2+}$ channels operate in a subthreshold voltage range, with an overlap between steady-state activation and inactivation curves that produces a voltage range where a subset of T-type channels are constitutively open (i.e., steady-state current, $I_{\text {stst }}$ or "window current") (Hughes et al., 1999; Crunelli et al., 2005). Furthermore, we observed that the leptin-mediated depolarization of RMP induced a voltage right-shift into the active T-type window current range, resulting in an increase of the steady state T-type calcium current from 40 to $70 \%$; which ultimately affects the intrinsic excitability of POMC neurons. This study was focused on the somatic response of POMC cultured neurons from newborn mice, thus it did not address questions pertaining to the hypothalamic circuit level. However, our results show T-type channel activity is necessary for leptin-mediated effects on hypothalamic POMC neuron excitability and confirms T-type channels as possible additional drug targets for leptinmediated functions, such as metabolic energy regulation and control of food satiety.

\section{DATA AVAILABILITY STATEMENT}

The raw data supporting the conclusions of this article will be made available by the authors, without undue reservation. A previous version of this research is available (https://www. biorxiv.org/content/10.1101/2020.07.21.214296v1). 


\section{ETHICS STATEMENT}

The animal protocols used in this study were reviewed and approved by an independent Institutional Animal Care and Use Committee at Loyola University Chicago (IACUC 2016032).

\section{AUTHOR CONTRIBUTIONS}

EP-R did the conception of research, approved the final version of the manuscript, and supervised the experiments. EP-R, EM-H, and PP designed the experiments, performed experiments, analyzed the data, interpreted results of experiments, and prepared the figures. EP-R and PP wrote the manuscript, edited,

\section{REFERENCES}

Ahima, R. S., and Flier, J. S. (2000). Leptin. Annu. Rev. Physiol. 62, 413-437. doi: 10.1146/annurev.physiol.62.1.413

Ahima, R. S., Prabakaran, D., Mantzoros, C., Qu, D., Lowell, B., Maratos-Flier, E., et al. (1996). Role of leptin in the neuroendocrine response to fasting. Nature 382, 250-252. doi: 10.1038/382250a0

Ambudkar, I. S. (2006). Ca2+ signaling microdomains: platforms for the assembly andregulation of TRPC channels. Trends Pharmacol. Sci. 27, 25-32. doi: 10. 1016/j.tips.2005.11.008

Anderson, D., Mehaffey, W. H., Iftinca, M., Rehak, R., Engbers, J. D., Hameed, S., et al. (2010). Regulation of neuronal activity by Cav3-Kv4 channel signaling complexes. Nat. Neurosci. 13, 333-337. doi: 10.1038/nn.2493

Balasubramanyan, S., Stemkowski, P. L., Stebbing, M. J., and Smith, P. A. (2006). Sciatic chronic constriction injury produces cell-type-specific changes in the electrophysiological properties of rat substantia gelatinosa neurons. J. Neurophysiol. 96, 579-590. doi: 10.1152/jn.00087.2006

Baver, S. B., Hope, K., Guyot, S., Bjorbaek, C., Kaczorowski, C., and O’Connell, K. M. (2014). Leptin modulates the intrinsic excitability of AgRP/NPY neurons in the arcuate nucleus of the hypothalamus. J. Neurosci. 34, 5486-5496. doi: 10.1523/jneurosci.4861-12.2014

Bean, B. P. (1985). Two kinds of calcium channels in canine atrial cells. differences in kinetics, selectivity, and pharmacology. J. Gen. Physiol. 86, 1-30. doi: 10.1085/ jgp.86.1.1

Bijlenga, P., Liu, J.-H., Espinos, E., Haenggeli, C.-A., Fischer-Lougheed, J., Bader, C. R., et al. (2000). T-type $\alpha 1 \mathrm{H} \mathrm{Ca} 2+$ channels are involved in $\mathrm{Ca} 2+$ signaling during terminal differentiation (fusion) of human myoblasts. Proc. Natl. Acad. Sci. U.S.A. 97, 7627-7632. doi: 10.1073/pnas.97.13.7627

Bosch, M. A., Hou, J., Fang, Y., Kelly, M. J., and Ronnekleiv, O. K. (2009). 17Beta-estradiol regulation of the mRNA expression of T-type calcium channel subunits: role of estrogen receptor alpha and estrogen receptor beta. J. Comp. Neurol. 512, 347-358. doi: 10.1002/cne.21901

Chemin, J., Monteil, A., Perez-Reyes, E., Nargeot, J., and Lory, P. (2001). Direct inhibition of T-type calcium channels by the endogenous cannabinoid anandamide. EMBO J. 20, 7033-7040. doi: 10.1093/emboj/20.24. 7033

Chorvat, R. J. (2013). Peripherally restricted CB1 receptor blockers. Bioorg. Med. Chem. Lett. 23, 4751-4760. doi: 10.1016/j.bmcl.2013.06.066

Cookson, J., and Duffett, R. (1998). Fluoxetine: therapeutic and undesirable effects. Hosp. Med. 59, 622-626.

Cowley, M. A., Smart, J. L., Rubinstein, M., Cerdan, M. G., Diano, S., Horvath, T. L., et al. (2001). Leptin activates anorexigenic POMC neurons through a neural network in the arcuate nucleus. Nature 411, 480-484. doi: 10.1038/35078085

Crunelli, V., Tóth, T. I., Cope, D. W., Blethyn, K., and Hughes, S. W. (2005). The 'window' T-type calcium current in brain dynamics of different behavioural states. J. Physiol. 562, 121-129. doi: 10.1113/jphysiol.2004.076273

Domingos, A. I., Vaynshteyn, J., Voss, H. U., Ren, X., Gradinaru, V., Zang, F., et al. (2011). Leptin regulates the reward value of nutrient. Nat. Neurosci. 14, 1562-1568. doi: 10.1038/nn.2977 and revised the manuscript. All authors contributed to the article and approved the submitted version.

\section{FUNDING}

This article was based upon work supported by the National Science Foundation under grant no. 1022075 (EP-R).

\section{ACKNOWLEDGMENTS}

We thank all members of the Piedras Laboratory, Drs. O'Connell and Don Carlos for helpful input and comments.

Farr, O. M., Sloan, D. M., Keane, T. M., and Mantzoros, C. S. (2014). Stressand PTSD-associated obesity and metabolic dysfunction: a growing problem requiring further research and novel treatments. Metabolism 63, 1463-1468. doi: 10.1016/j.metabol.2014.08.009

Friedman, J. M. (2004). Modern science versus the stigma of obesity. Nat. Med. 10, 563-569. doi: 10.1038/nm0604-563

Gao, Y., Yao, T., Deng, Z., Sohn, J.-W., Sun, J., Huang, Y., et al. (2017). TrpC5 mediates acute leptin and serotonin effects via Pomc neurons. Cell Rep. 18, 583-592. doi: 10.1016/j.celrep.2016.12.072

Gutierrez, C., Cox, C. L., Rinzel, J., and Sherman, S. M. (2001). Dynamics of lowthreshold spike activation in relay neurons of the cat lateral geniculate nucleus. J. Neurosci. 21, 1022-1032. doi: 10.1523/jneurosci.21-03-01022.2001

Harvey, J. (2007). Leptin regulation of neuronal excitability and cognitive function. Curr. Opin. Pharmacol. 7, 643-647. doi: 10.1016/j.coph.2007.10.006

Harvey, J., and Ashford, M. L. (2003). Leptin in the CNS: much more than a satiety signal. Neuropharmacology 44, 845-854. doi: 10.1016/S0028-3908(03)00076-5

Haynes, W. G., Morgan, D. A., Djalali, A., Sivitz, W. I., and Mark, A. L. (1999). Interactions between the melanocortin system and leptin in control of sympathetic nerve traffic. Hypertension 33(1 Pt 2), 542-547. doi: 10.1161/01. HYP.33.1.542

Hughes, S. W., Cope, D. W., Toth, T. I., Williams, S. R., and Crunelli, V. (1999). All thalamocortical neurones possess a T-type $\mathrm{Ca} 2+$ 'window'current that enables the expression of bistability-mediated activities. J. Physiol. 517, 805-815. doi: 10.1111/j.1469-7793.1999.0805s.x

Huguenard, J. (1996). Low-threshold calcium currents in central nervous system neurons. Annu. Rev. Physiol. 58, 329-348. doi: 10.1146/annurev.ph.58.030196. 001553

Kenny, P. J. (2011). Common cellular and molecular mechanisms in obesity and drug addiction. Nat. Rev. Neurosci. 12, 638-651. doi: 10.1038/nrn3105

Kim, S., and Moustaid-Moussa, N. (2000). Secretory, endocrine and autocrine/paracrine function of the adipocyte. J. Nutr. 130, 3110S-3115S.

Lambert, R. C., Bessaïh, T., Crunelli, V., and Leresche, N. (2014). The many faces of T-type calcium channels. Pflügers Arch. 466, 415-423. doi: 10.1007/s00424013-1353-6

Llinás, R. R. (1988). The intrinsic electrophysiological properties of mammalian neurons: insights into central nervous system function. Science 242, 1654-1664. doi: 10.1126/science.3059497

Lu, V. B., Moran, T. D., Balasubramanyan, S., Alier, K. A., Dryden, W. F., Colmers, W. F., et al. (2006). Substantia gelatinosa neurons in defined-medium organotypic slice culture are similar to those in acute slices from young adult rats. Pain 121, 261-275. doi: 10.1016/j.pain.2006.01.009

Mlinar, B., and Enyeart, J. (1993). Block of current through T-type calcium channels by trivalent metal cations and nickel in neural rat and human cells. J. Physiol. 469, 639-652. doi: 10.1113/jphysiol.1993.sp019835

Myers, M. G., Cowley, M. A., and Munzberg, H. (2008). Mechanisms of leptin action and leptin resistance. Annu. Rev. Physiol. 70, 537-556. doi: 10.1146/ annurev.physiol.70.113006.100707

Perez-Reyes, E. (2003). ). Molecular physiology of low-voltage-activated t-type calcium channels. Physiol. Rev. 83, 117-161. doi: 10.1152/physrev.00018.2002 
Perissinotti, P. P., Ethington, E. G., Almazan, E., Martínez-Hernández, E., Kalil, J., Koob, M. D., et al. (2015). Calcium current homeostasis and synaptic deficits in hippocampal neurons from Kelch-like 1 knockout mice. Front. Cell. Neurosci. 8:444. doi: 10.3389/fncel.2014.00444

Perissinotti, P. P., Ethington, E. G., Cribbs, L., Koob, M. D., Martin, J., and PiedrasRenteria, E. S. (2014). Down-regulation of endogenous KLHL1 decreases voltage-gated calcium current density. Cell Calcium 55, 269-280. doi: 10.1016/ j.ceca.2014.03.002

Perissinotti, P. P., Rivero-Echeto, M. C., Garcia-Rill, E., Bisagno, V., and Urbano, F. J. (2018). Leptin alters somatosensory thalamic networks by decreasing gaba release from reticular thalamic nucleus and action potential frequency at ventrobasal neurons. Brain Struct. Funct. 223, 2499-2514. doi: 10.1007/s00429018-1645-x

Qiu, J., Bosch, M. A., Jamali, K., Xue, C., Kelly, M. J., and Rønnekleiv, O. K. (2006). Estrogen upregulates T-type calcium channels in the hypothalamus and pituitary. J. Neurosci. 26, 11072-11082. doi: 10.1523/jneurosci.3229-06.2006

Qiu, J., Bosch, M. A., Meza, C., Navarro, U.-V., Nestor, C. C., Wagner, E. J., et al. (2018). Estradiol protects proopiomelanocortin neurons against insulin resistance. Endocrinology 159, 647-664. doi: 10.1210/en.2017-00793

Qiu, J., Fang, Y., Bosch, M. A., Ronnekleiv, O. K., and Kelly, M. J. (2011). Guinea pig kisspeptin neurons are depolarized by leptin via activation of TRPC channels. Endocrinology 152, 1503-1514. doi: 10.1210/en.20101285

Qiu, J., Fang, Y., Ronnekleiv, O. K., and Kelly, M. J. (2010). Leptin excites proopiomelanocortin neurons via activation of TRPC channels. J. Neurosci. 30, 1560-1565. doi: 10.1523/jneurosci.4816-09.2010

Qiu, J., Zhang, C., Borgquist, A., Nestor, C. C., Smith, A. W., Bosch, M. A., et al. (2014). Insulin excites anorexigenic proopiomelanocortin neurons via activation of canonical transient receptor potential channels. Cell Metab. 19, 682-693. doi: 10.1016/j.cmet.2014.03.004

Rehak, R., Bartoletti, T. M., Engbers, J. D., Berecki, G., Turner, R. W., and Zamponi, G. W. (2013). Low voltage activation of KCa1.1 current by Cav3-KCa1.1 complexes. PLoS One 8:e61844. doi: 10.1371/journal.pone.0061844

Rivero-Echeto, M. C., Perissinotti, P. P., González-Inchauspe, C., Kargieman, L., Bisagno, V., and Urbano, F. J. (2020). Simultaneous administration of cocaine and caffeine dysregulates HCN and T-type channels. Psychopharmacology 238, 787-810. doi: 10.1007/s00213-020-05731-5

Robitaille, R., Garcia, M. L., Kaczorowski, G. J., and Chariton, M. P. (1993). Functional colocalization of calcium and calcium-gated potassium channels in control of transmitter release. Neuron 11, 645-655. doi: 10.1016/0896-6273(93) 90076-4

Sabourin, J., Robin, E., and Raddatz, E. (2011). A key role of TRPC channels in the regulation of electromechanical activity of the developing heart. Cardiovasc. Res. 92, 226-236. doi: 10.1093/cvr/cvr167

Schlick, B., Flucher, B. E., and Obermair, G. J. (2010). Voltage-activated calcium channel expression profiles in mouse brain and cultured hippocampal neurons. Neuroscience 167, 786-798. doi: 10.1016/j.neuroscience.2010. 02.037

Schneider, C. A., Rasband, W. S., and Eliceiri, K. W. (2012). NIH Image to ImageJ: 25 years of image analysis. Nat. Methods 9, 671-675. doi: 10.1038/nmeth.2089

Schwartz, M. W., Woods, S. C., Porte, D. Jr., Seeley, R. J., and Baskin, D. G. (2000). Central nervous system control of food intake. Nature 404, 661-671. doi: $10.1038 / 35007534$
Shioda, S., Funahashi, H., Nakajo, S., Yada, T., Maruta, O., and Nakai, Y. (1998). Immunohistochemical localization of leptin receptor in the rat brain. Neurosci. Lett. 243, 41-44. doi: 10.1016/S0304-3940(98)00082-2

Sohn, J.-W., Xu, Y., Jones, J. E., Wickman, K., Williams, K. W., and Elmquist, J. K. (2011). Serotonin 2C receptor activates a distinct population of arcuate pro-opiomelanocortin neurons via TRPC channels. Neuron 71, 488-497. doi: 10.1016/j.neuron.2011.06.012

Strübing, C., Krapivinsky, G., Krapivinsky, L., and Clapham, D. E. (2001). TRPC1 and TRPC5 form a novel cation channel in mammalian brain. Neuron 29, 645-655. doi: 10.1016/s0896-6273(01)00240-9

Takekoshi, K., Ishii, K., Nanmoku, T., Shibuya, S., Kawakami, Y., Isobe, K., et al. (2001). Leptin stimulates catecholamine synthesis in a PKC-dependent manner in cultured porcine adrenal medullary chromaffin cells. Endocrinology 142, 4861-4871. doi: 10.1210/endo.142.11.8484

Traboulsie, A., Chemin, J., Kupfer, E., Nargeot, J., and Lory, P. (2006). T-type calcium channels are inhibited by fluoxetine and its metabolite norfluoxetine. Mol. Pharmacol. 69, 1963-1968. doi: 10.1124/mol.105.020842

Uebele, V. N., Gotter, A. L., Nuss, C. E., Kraus, R. L., Doran, S. M., Garson, S. L., et al. (2009). Antagonism of T-type calcium channels inhibits high-fat diet-induced weight gain in mice. J. Clin. Invest. 119, 1659-1667. doi: 10.1172/ jci36954

van den Top, M., Lee, K., Whyment, A. D., Blanks, A. M., and Spanswick, D. (2004). Orexigen-sensitive NPY/AgRP pacemaker neurons in the hypothalamic arcuate nucleus. Nat. Neurosci. 7, 493-494. doi: 10.1038/nn1226

Vivas, O., Moreno, C. M., Santana, L. F., and Hille, B. (2017). Proximal clustering between BK and CaV1. 3 channels promotes functional coupling and BK channel activation at low voltage. Elife 6:e28029.

Wang, J.-H., Wang, F., Yang, M.-J., Yu, D.-F., Wu, W.-N., Liu, J., et al. (2008). Leptin regulated calcium channels of neuropeptide $\mathrm{Y}$ and proopiomelanocortin neurons by activation of different signal pathways. Neuroscience 156, 89-98. doi: 10.1016/j.neuroscience.2008.04.079

Wilfong, A. A., and Willmore, L. J. (2006). Zonisamide - a review of experience and use in partial seizures. Neuropsychiatr. Dis. Treat. 2, 269-280. doi: 10.2147/ nedt.2006.2.3.269

Williams, K. W., and Elmquist, J. K. (2012). From neuroanatomy to behavior: central integration of peripheral signals regulating feeding behavior. Nat. Neurosci. 15, 1350-1355. doi: 10.1038/nn.3217

Zhang, C., Bosch, M. A., Rick, E. A., Kelly, M. J., and Rønnekleiv, O. K. (2009). $17 \beta$-estradiol regulation of T-type calcium channels in gonadotropin-releasing hormone neurons. J. Neurosci. 29, 10552-10562. doi: 10.1523/jneurosci.296209.2009

Conflict of Interest: The authors declare that the research was conducted in the absence of any commercial or financial relationships that could be construed as a potential conflict of interest.

Copyright (c) 2021 Perissinotti, Martínez-Hernández and Piedras-Rentería. This is an open-access article distributed under the terms of the Creative Commons Attribution License (CC BY). The use, distribution or reproduction in other forums is permitted, provided the original author(s) and the copyright owner(s) are credited and that the original publication in this journal is cited, in accordance with accepted academic practice. No use, distribution or reproduction is permitted which does not comply with these terms. 